\title{
The numerical analysis of dynamically loaded ceramic: a crack softening approach
}

\author{
Paul J Hazell and Michael J Iremonger \\ Engineering Systems Department, Cranfield University, RMCS, Shrivenham, Swindon, SN6 8LA, UK
}

\section{Summary}

A physically based crack softening approach to modelling the failure of brittle materials that have been subjected to dynamic loading is presented and applied to a two-dimensional non-linear transient dynamic hydrocode. It is assumed that there are a number of evenly distributed and orientated microflaws within the brittle material that are activated by a dynamically applied stress. The mode I and mode II stress intensity factors are calculated and compared to critical values, at which point the cracks grow at a velocity dependent on the mode I stress intensity factor. The strength of the ceramic is degraded according to the length of the cracks. A simulation of a steel sphere impacting and penetrating a ceramic target at $1500 \mathrm{~m} / \mathrm{s}$ is presented. Comparisons are drawn from experimental data.

Keywords: crack-softening, hydrocode, dynamic, failure, ceramic, strength-loss.

\subsection{Introduction}

Under high rate dynamic loading conditions, standard numerical techniques and packages are inappropriate to simulate the response of materials. This has led to the development of non-linear transient dynamic computer codes (hydrocodes) where the conservation equations of mass, momentum and energy are solved simultaneously 
with an equation of state and constitutive relationship. The equation of state describes the compressibility effects and irreversible thermodynamic processes that generally occur during high strain rate events. It relates the density (or volume) and the internal energy with the material (or temperature) with pressure. The constitutive relationship describes the nature of the material by relating the stress in the material with the strain (or distortion) necessary to produce this stress.

Hydrocodes were initially developed to investigate hypervelocity impact phenomena where hydrodynamic pressure dominates the behaviour of colliding solids and strength effects can be ignored. The presence of shock waves and localised material response led to the development of the above mentioned complex constitutive relationships and equations of state. Hydrocodes have also demonstrated their usefulness in lower strain-rate applications where strength of materials effects are important.

Under dynamic compressive loading, brittle materials generally deform inelastically. The inelastic response of these brittle materials is usually attributed to the nucleation, growth and coalescence of microcracks. Early work $^{1}$ has shown that failure of polycrystalline alumina is controlled by microplasticity, i.e. twinning and possibly slip, which nucleate grain boundary cracks. The nucleation, growth and coalescence of these cracks causes extensive stiffness loss and strength degradation within the material $^{2}$.

Traditionally, failure of a brittle material has been modelled in a hydrocode by a complete loss of material strength once failure is initiated, though it is now common 
to use a 'cumulative damage' algorithm to simulate failure and strength degradation of the material. This would normally be achieved by the calculation of a scalar damage parameter that is related to the plastic strain within the material. Such a method does not relate closely to the physical crack nucleation and tensile crack growth that one would expect on dynamic loading.

There is, therefore, a requirement for detailed crack softening models to be developed and implemented in such hydrocodes as AUTODYN ${ }^{3}$. It is the authors' intention to present and discuss a fracture mechanics based crack softening model that can be used in conjunction with a hydrocode. The principles of this model has been presented by Hazell and Iremonger ${ }^{4}$, however this model did not take into account plasticity effects. This new adaptation takes into account brittle failure in compression and tension and failure by plastic deformation. In addition, the approach to the strength degradation of brittle materials has been enhanced to provide a more realistic physical response to dynamic failure. An analysis of a steel sphere impacting a ceramic block is presented.

\subsection{Modelling the failure of brittle materials}

Due to the brittle nature of ceramics, a fracture mechanics based model seems plausible for simulating dynamic failure because it provides a sound theoretical basis for analysing the cracking process. Several models have been developed to simulate the quasi-static brittle behaviour of rocks and therefore their application can be extended to include the modelling of ceramic. 
Margolin $^{5}$ developed a model to simulate the dynamic failure processes that occur in rocks under compression and tension. This microphysical model considers crack opening and sliding under both compressive and tensile loading. The effects of strain rate, pressure and damage on compressive strength are evaluated in the model. Costin and Stone ${ }^{6}$ considered microcrack orientation and anisotropic growth in different directions. The damage is defined as a vector whose components are in 13 different orientations in two-dimensional problems and 144 directions in three-dimensional problems. The effect of confining pressure, crack interaction and dilatancy are taken into account for quasi-static problems in both compression and tension. The constitutive model of Horrii and Nemat-Nasser ${ }^{7}$ is based on the elastic moduli of cracked bodies. The effect of crack opening, sliding and tearing is analytically derived. The model treats the damage as the relative volume of microcracks and is considered as a single scalar, independent of microcrack orientation. Microcrack growth is not considered, instead this model was developed to calculate the elastic moduli of cracked bodies for which the average number of cracks per volume and the average crack size are known. Krajcinovic and Fanella ${ }^{8}$ described the non-linear behaviour of brittle concrete under static loading using continuum based constitutive relationships. A scalar damage parameter is calculated for each phase of crack growth. Effects of confining pressure and dilatancy are also modelled. Ilankamban and Krajcinovic ${ }^{9}$ modelled the non-linear behaviour of progressively deteriorating brittle materials under static loading. In this model, damage is irreversible and is expressed through an empirical relationship between the microcrack densities and their conjugate thermodynamic forces. The effects of confining pressure, dilatancy and anisotropy are also simulated. 
Various models have been suggested for modelling the response of ceramics under dynamic loading. Ravichandran and Subhash ${ }^{10}$ and Deng and Nemat-Nasser ${ }^{11}$ used a sliding crack model ${ }^{12}$ as a basis for modelling the failure of brittle materials under dynamic compressive loading. Addesio and Johnson $^{13}$ presented a microphysical model to describe the complex behaviour of ceramics under dynamic loading. Both crack opening due to tension and crack sliding due to compression were modelled. Rajendran ${ }^{14}$ has successfully modelled the inelastic response of alumina using a “calibrated” approach where the physical parameters were matched with low velocity plate impact results. All of these authors have presented some verification of their approaches to modelling the impact of brittle materials. However, most of the papers do not contain pictures showing brittle failure caused by dynamic impact. Instead, the results are restricted to depths of penetration or plate impact data.

Recently, other techniques have been developed that are particularly useful in the modelling of failure in brittle materials. Smooth Particle Hydrodynamics (SPH) is a gridless approach where all mass is 'lumped' into individual particles that are usually regularly spaced ${ }^{15}$. Interaction between the particles is defined by a kernel function that exerts influence in a small neighbourhood around each particle. SPH has a number of advantages over other methods of processing in that it is gridless and therefore there are no mesh tangling problems, as in the standard Lagrange methodology (which uses mesh points that are embedded in the material and move with it in space). Particles are required only where there is material, and fracture and ejection of material can be readily modelled by the separation of particles. However, SPH as a method for penetration studies, has not yet been fully developed. Energy errors and 'numerical fracture' still cause problems although the method has shown 
some measure of success ${ }^{16}$. Rockfield Software has developed a finite / discrete element code that has recently shown some promise in the analysis of penetration into ceramic by a long $\operatorname{rod}^{17}$. Initially, the target in question is modelled as a continuum using finite element methods. Using simple constitutive relationships and fracture algorithms, the code is able to accurately predict fracture initiation. Progressive fracturing of the continuum results in the formation of discrete elements which may consist of one or more deformable finite elements or even parts of an original element. This process requires re-meshing algorithms to convert fractured zones into discrete element representation. Therefore, the formation of individual fragments can be readily simulated.

\subsection{The Model}

\subsection{Dynamic Failure in Compression}

Most brittle solids contain inhomogeneities such as small holes, cracks or phases which have different moduli or strengths from those of the matrix. When a brittle material is subjected to a large confining stress, any of these inhomogeneities can act as nuclei for new cracks. These microcracks eventually coalesce to cause axial splitting.

For a straight slanted flaw that is loaded under biaxial compression, where $\sigma_{1}>\sigma_{2}$, tensile cracks nucleate from the tips of the flaw and grow in the direction of maximum compression ${ }^{18,19}$. The tension cracks (or 'winglets') are assumed to grow so as to maximise the mode $I$ stress intensity factor $K_{I}$ (Figure 1). The growing 'winglets' are approximated by straight cracks because the sigmoidal cracks grow to 
several times the initial flaw length ${ }^{10,20}$. Under compression, the failure depends on the relative magnitude of the principal stresses. The kinked tension cracks are approximated by a single large crack of length $2 l$ that grows in the direction of maximum compression. The analogy can also be applied to a crack emanating from a circular pore ${ }^{21}$.

Figure 1: Idealised unit cell model for a sliding crack under biaxial compression.

The mode I and mode II stress intensity factors $K_{I}$ and $K_{I I}$ at the tip of each of the tension cracks shown in Figure 1 and under biaxial compressive loading are given by ${ }^{4}$

$$
K_{I}=\frac{T \cos \theta}{\sqrt{\left[\pi\left(l+l^{*}\right)\right]}}-\sigma_{2} \sqrt{(\pi l)} \text { and } K_{I I}=\frac{-T \sin \theta}{\sqrt{\left[\pi\left(l+l^{*}\right)\right]}} \text {. }
$$

where $T \cos \theta$ and $T \sin \theta$ are the crack opening and sliding loads respectively. In this instance, the compressive stresses are assumed positive.

These results give good estimates of the stress intensity when $l$ is large but an unstability occurs when the length $l$ becomes vanishingly small. To overcome errors in the calculation of $K_{I}$ and $K_{I I}$ when the length of the tension cracks are extremely small, a constant $\left(l^{*}\right)$ is added in order to produce an 'effective' crack length $\left(l+l^{*}\right)$ which is dependant on the initial flaw size $e^{20} . l^{*}$ has been estimated ${ }^{7}$ to be $0.27 a$.

The above theory calculates the stress intensity factors for a material that is subjected to far field stresses that create shear stress along the surface of an inclined crack. It 
does not however predict the nucleation of a flaw under large hydrostatic stress. Therefore, to simulate the effect of 'crushing' or 'pulverising' of the material ahead of the penetrator, a relationship was used which evaluates the degree of plasticity within the material.

'Plasticity' in ceramics has been observed by a number of authors ${ }^{22,23}$ and is generally exhibited by the process of twinning and/or slip within the crystalline structure. Twinning and slip within individual grains and plasticity within interfacial materials give rise to the nucleation of intergranular microcracks, which eventually coalesce causing failure of the material.

Clearly, this physical process is practically impossible to simulate accurately using a Lagrangian hydrocode. To overcome this problem, an 'effective plastic strain' failure criterion was used similar to that used by Persson $^{24}$ and implemented within the current version of AUTODYN ${ }^{\mathrm{TM}}{ }^{3}$.

Damage in a cell is initiated when a minimum degree of plasticity $\left(\varepsilon_{P 1}\right)$ is observed. This value is assumed to be the effective plasticity required to nucleate inter-granular cracks. A limiting value of effective plastic strain $\left(\varepsilon_{P 2}\right)$ is set which determines the point at which complete coalescence and failure has occurred within the individual cell. This relationship is shown schematically below in Figure 2.

Figure 2: Relationship between the effective plastic strain and the damage accumulated within a Lagrangian cell ( $D=1$ represents the intact material, $D=0$ represents failed material). 
Once the material has failed completely (due to the coalescence of the plasticitynucleated microcracks) no further damage can be accumulated.

\subsection{Dynamic Failure in Tension}

Much of the failure observed in a brittle material subjected by a dynamic load is tensile. Hoop stresses induced by the radial movement of the material due to penetration are sufficient to nucleate tensile flaws which eventually coalesce and cause failure. Moreover, tensile spall planes are generated by tensile waves reflected from free surfaces and interact with inhomogeneities to nucleate microcracks. When a tensile pulse passes a microcrack within a brittle material the mode I and mode II stress intensities depend on the time of duration and the speed of the pulse.

Assuming that the tensile pulse arrives uniformly at the crack tip, the stress intensity factors can be described ${ }^{25}$ by

$$
K_{I}(t)=C_{I} \sigma_{\theta} \sqrt{2 \pi c_{l} t} \text { and } K_{I I}(t)=C_{I I} \tau_{\theta} \sqrt{2 \pi c_{l} t} \text {, }
$$

where $\sigma_{\theta}$ and $\tau_{\theta}$ are the resolved normal and shear stresses acting on the microcrack, $c_{l}$ and $c_{s}$ are the longitudinal and shear wave velocities and $t$ is time. $\mathrm{C}_{\mathrm{I}}$ and $\mathrm{C}_{\mathrm{II}}$ are material constants:

$$
C_{I}=\frac{\sqrt{2(1-v)}}{\pi(1-v)} \text { and } C_{I I}=\frac{2}{\pi} \sqrt{\frac{2 c_{s}}{(1-v) c_{l}}}
$$

where $v$ is the Poisson's ratio of the material. 


\subsection{Crack Distribution}

It is assumed that there is a uniformly distributed number of microcracks of even length distributed through the target material. For simplicity, it is assumed that there is one orientated microcrack per cell, evenly distributed as shown in Figure 3. However, it is simple to apply a random Weibull distribution function to the crack position and to vary the length in a random fashion. It is assumed that residual stresses within the material are minimal.

Figure 3: Initial distribution of flaws in cells of height $\mathrm{h}$ and width $\mathrm{w}$.

Although the orientations of microflaws are generally random, failure occurs from cracks that nucleate from microflaws of some particular angle. For two-dimensional analysis, the angle which is chosen here is that suggested by Nemat-Nasser and Deng $^{26}$, namely $\theta=\pi / 5$. The length of the initial microcrack is taken to be the average size of a microflaw within the material.

\section{4 Crack Initiation}

In conventional static Linear Elastic Fracture Mechanics, the onset of crack growth occurs when the stress intensity factor measured at the crack tip exceeds some critical value $K_{c}$. This value is often referred to as the static fracture toughness.

In elastodynamic fracture, the onset of growth of a rapidly loaded stationary crack is given by

$$
K(t, 0)=K_{d}(\dot{\sigma})
$$


where $K_{d}$ is the dynamic initiation toughness which may also depend on the temperature.

It has been shown experimentally ${ }^{27}$ that

$$
\frac{K_{d}}{K_{c}}=\alpha,
$$

where $\alpha$ is a constant. At initiation, $\alpha$ varies between $1.25-1.40$ for ceramics ${ }^{28}$. According to the criterion given above, a propagating crack will be arrested when the stress intensity factor becomes smaller than or equal to a critical value.

This can be expressed as

$$
K \leq K_{D}(0) \equiv K_{I a}^{d y n}
$$

where $K_{I a}^{d y n}$ denotes the dynamic crack arrest toughness.

Shockey, Kalthoff and Erlick ${ }^{29}$ reported a substantial reduction of the crack initiation toughness in brittle epoxy due to dynamic loading. The estimate for the fracture toughness under dynamic loading conditions imposed was about $K_{I d}=0.72 \mathrm{MPa} \sqrt{m}$ compared to the slow moving fracture toughness of $K_{I c}=1.1 \mathrm{MPa} \sqrt{m}$. For ceramics however, the opposite seems to occur. Yang and Kobayashi ${ }^{30}$ noted an increase in initiation toughness under dynamic loading conditions. Unlike metals and polymers, 
they observed that the dynamic crack arrest toughness did not exist i.e. once crack growth was initiated, stable crack growth was observed even when the stress intensity was lowered.

The energy release rate for a stationary microcrack affected by a static and transient load (plane strain) is given ${ }^{25}$ by

$$
G=\frac{1-v^{2}}{E}\left(K_{I}^{2}+K_{I I}^{2}\right)
$$

where $E$ represents the Young's modulus of the material.

To calculate the energy release rate of the crack, for both compression and tension, the respective mode I and mode II stress intensity factors are substituted into Equation 7. This then is checked against a critical energy release rate $G_{c}$.

Because the mode I and mode II stress intensities in compression are calculated from Linear Elastic Fracture Mechanics, we must consider the effect of loading the specimen dynamically. We do this by introducing the relationship:

$$
\frac{G_{d}}{G}=h
$$

where $G_{d}$ is the dynamic energy release rate, $G$ is the static energy release rate calculated from Equation 7 and $h$ is a constant to be calculated from experimental 
data. Both the dynamic and static critical energy release rate (plane strain) for tensile crack initiation can be calculated using the relationship:

$$
G_{c}=\frac{\left(1-v^{2}\right)}{E} K_{I c}
$$

Table 1 shows the comparison of the static and the dynamic fracture toughness and their corresponding critical energy release rates for two grades of alumina, Coors AD995 and AD998. The average value of $h$ from the three references for the two grades of alumina is 1.69. No data is currently available for Sintox-FA.

The modified value of the energy release rate is compared with the critical energy release rate for crack initiation:

$$
G_{d}=G_{c}
$$

Table 1: Comparison of dynamic and static fracture toughness values for alumina and their corresponding critical energy release rates.

\begin{tabular}{cccccccc}
\hline Ref. & $\begin{array}{c}K_{I c} \\
(\mathrm{MPa} \sqrt{\mathrm{m}})\end{array}$ & $\begin{array}{c}K_{I d} \\
(\mathrm{MPa} \sqrt{\mathrm{m}})\end{array}$ & $\begin{array}{c}\alpha \\
\left(K_{I d} / K_{I c}\right)\end{array}$ & $\begin{array}{c}G_{c} \\
\left(\mathrm{~J} / \mathrm{m}^{2}\right)\end{array}$ & $\begin{array}{c}G_{d} \\
\left(\mathrm{~J} / \mathrm{m}^{2}\right)\end{array}$ & $\begin{array}{c}h \\
\left(\mathrm{G}_{\mathrm{d}} / \mathrm{G}_{\mathrm{c}}\right)\end{array}$ & $\begin{array}{c}\text { Alumina } \\
(\text { Coors })\end{array}$ \\
\hline$[28]$ & 2.7 & 3.5 & 1.30 & 20.0 & 33.7 & 1.69 & AD998 \\
{$[30]$} & 4.3 & 5.7 & 1.33 & 46.0 & 80.7 & 1.75 & AD995 \\
{$[32]$} & 2.9 & 3.7 & 1.28 & 23.1 & 37.7 & 1.63 & AD998 \\
\hline
\end{tabular}

In tension however, the mode I and mode II stress intensity factors are calculated from the laws of elastodynamic fracture mechanics and a simple initiation law can therefore be used: 


$$
G=G_{c}
$$

\subsection{Crack Propagation Velocity}

Once a crack has initiated, it will propagate at a velocity dependent on the applied stress intensity ${ }^{31}$. Under compression, the crack will propagate in a stable manner; under tension the crack will propagate catastrophically at a velocity (i) fast approaching the Rayleigh wave speed $\left(c_{R}\right)$ of the material. Failure in compression and in tension is described by

$$
i=c_{R}\left(1-\frac{K_{d}}{K_{I}}\right) .
$$

Although $K_{d}$ is a function of the rate of applied stress for most materials little evidence exists to support this for ceramics ${ }^{32}$. In this instance, $K_{d}$ is assumed constant and can be calculated by substituting a value of the critical stress intensity factor for a ceramic $(4.60 \mathrm{MPa} \sqrt{ } \mathrm{m})$ and the values of $\alpha$ in Table 1 into Equation 5.

\subsection{Degradation of the Material Properties}

To simulate the degradation of the stiffness and strength which occurs due to the nucleation, growth and coalescence of microcracks, the proposed model of Fenghui et $a l^{33}$ was adopted. Fenghui and his colleagues developed a model that took into account the stiffness degradation due to the presence of porosity and extended it to 
correlate flexural strength with the presence of cavities. The model was validated using published data for the flexural strength of alumina and silicon nitride.

The relation between Young's modulus and porosity is given by

$$
E=E_{0} \frac{1-P^{o}}{1+\beta P^{o}},
$$

where $E$ and $E_{0}$ denote the Young's modulus of the porous material and pore-free material, respectively. $\beta$ is a pore geometry factor which is determined by pore shape and $P^{o}$ is the degree of porosity which is defined as follows:

$$
P^{o}=\frac{1}{A} \sum \pi a^{2}
$$

where $A$ is the representative area which contains the porosity and $a$ is the maximum radius of the holes. For simplicity, the holes are taken as circles ${ }^{33}$ and Equation (13) becomes

$$
E=E_{0} \frac{1-P^{o}}{1+2.5 P^{o}}
$$

In the coded user-subroutine, the degree of porosity is calculated by dividing the crack length by the maximum possible crack length that the cell can sustain. Therefore, a problem is independent of the mesh density. 
Coble and Kingery ${ }^{34}$ studied sintered alumina containing isolated pores and measured the elastic moduli of each sample. Samples with a porosity of 5 to $50 \%$ were manufactured by firing them together for comparable grain development. This eliminated any structural variability except porosity. A comparison between the stiffness degradation observed by Coble and his colleagues and the model proposed by Fenghui et al is shown below in Figure 4. The elastic moduli were measured in transverse bending on ground and polished rectangular samples.

Figure 4: Degradation of the elastic moduli with increasing porosity.

Coble and Kingery ${ }^{34}$ also observed that the shear modulus degraded in a similar fashion to the Young's modulus. Therefore, it is reasonable to assume that a single relationship can be used to describe the degradation of the elastic moduli. In compression however, the stiffness of the solid would not be expected to change.

The yield strength of the brittle material is degraded according to the calculated scalar damage parameter $(D)$. In tension, it is expected that complete strength (and stiffness) loss occurs when the material has failed (cracked). Therefore, in this case, the yield strength is reduced to zero. However, in compression, fractured ceramic has been shown to exhibit some strength ${ }^{35}$. A factor $(\psi)$ is introduced which represents a percentage of the compressive yield strength below which the strength of the material cannot be degraded. Therefore, in compression, the yield strength never reaches zero but always retains some strength. In compression, the yield strength is calculated according to 


$$
Y=D \times Y_{0},
$$

where $Y_{0}$ is the yield strength of the undamaged material. The yield strength of the damaged material is unable to drop below $Y_{\min }$ :

$$
Y_{\min }=\psi \times Y_{0} .
$$

Currently, the factor $\psi$ is assumed to be $5 \%$. This value is consistent with experiments conducted by Hallas ${ }^{35}$ on compressed powdered samples using a Rosand drop tower system. Further experiments are required to confirm this value either by conducting dynamic drop-tower tests or by using a Split Hopkinson Pressure Bar to test the compressive strength of pre-fractured ceramic. To date, problems that have been associated with these methods have been the ability to consistently fragment the ceramic to a specific fragment size whilst maintaining bulk cohesiveness.

\subsection{The Model Implementation and Validation}

\subsection{Code Implementation}

Through the modification and implementation of a coded user subroutine, the crack softening model was applied to the hydrocode AUTODYN-2D.

For each cycle, the mode I and mode II stress intensity factors (Equations 1 and 2) are calculated for a single, evenly distributed, pre-existing flaw. The size of this flaw was taken to be the average grain size of the material. From these, the energy release rate is calculated (Equation 7) and compared with the critical value (Equations 10 and 11). 
If this critical value is exceeded, the crack will grow at a calculated velocity (Equation 12). The cracks will grow when under compression or tension. It is assumed that the cell is unable to carry load when the crack has propagated through the entire cell and coalesces with a neighbouring microcrack. Each crack is able to propagate past the cell boundaries by simply adding the length exceeding the cell dimension to the neighbouring cracks in the surrounding cells. A scalar damage parameter varying between 1 and 0 is defined in accordance with crack growth, where 1 represents no crack growth and 0 represents complete coalescence and failure of the Lagrangian cell.

For each model run, a linear equation of state is used coupled with a Mohr-Coulomb type strength model that is implemented within the modified user-subroutine. The Linear Equation of State is defined as

$$
P=K \mu
$$

where $P$ is the pressure, $K$ is the bulk modulus and $\mu$ is the compression $\left(\rho / \rho_{0}-1\right)$. This equation of state is of use for small compressions and where thermodynamic effects are negligible. This equation of state takes into account the loss of stiffness that is evident from the impact of brittle materials.

For a complete yield curve, an approach can be taken to evaluate the material dynamic response by examining the published values of Hugoniot Elastic Limit (HEL) for a variety of ceramics. Coakley ${ }^{36}$ adopted this approach for modelling the penetration of long rods into confined alumina. The yield strength is calculated for a 
fully dense ceramic (e.g. Lucalox) and is taken as the limiting strength for intact material. The pressure at which this limiting value is reached is not very well defined but of the order of $25 \mathrm{GPa}^{36}$. A second point of the curve is taken from the HEL of the ceramic that is to be modelled. Finally, the quasi-static yield strength is measured at room temperature with little confining pressure. The tensile hydrostatic pressure is determined from plate impact experiments.

Figure 5: A pressure dependent yield strength relationship for alumina (after Ref.36).

The approach that was adopted by Coakley (Figure 5) is a reasonable method as high pressure has been shown to result in pore compaction ${ }^{37}$ and suppression of fracture ${ }^{38}$. Moreover, the existence of pores results in premature nucleation and growth of microfractures. Their presence is also likely to reduce the HEL of the material. Therefore, the dynamic yield strength of a high purity, low porosity, fully dense alumina can be assumed to be the limiting case for the yielding of an alumina under large hydrostatic stress.

In the absence of Hugoniot data for Sintox-FA and Sintox-CL, the pressure dependent yield strength relationship derived by Rosenberg for AD85 and Lucalox was used. Above a pressure of $6 \mathrm{GPa}$, the linear response is assumed to break down as the HEL for the fully dense Lucalox is reached (i.e. yielding occurs for the fully compacted material). The spall strength was assumed to be 0.3GPa, which is consistent with published data for similar aluminas ${ }^{39}$. 
Above the yield surface defined by the above piecewise relationship, all calculated stress deviators are set to zero.

\subsection{Steel Sphere Impact of 25mm thick Alumina blocks}

A steel sphere impacting a Sintox-FA (95\% pure alumina manufactured by Morgan Matroc Ltd.) ceramic block at $1500 \mathrm{~m} / \mathrm{s}$ was modelled in two dimensions using axial symmetry. The projectile was $6.35 \mathrm{~mm}$ in diameter and the target was $25 \mathrm{~mm}$ thick. A steel sphere was chosen because the material is homogeneous and well characterised. The initial mesh density of both the target and the projectile was $0.5 \mathrm{~mm}$ (i.e. each cell was $0.5 \times 0.5 \mathrm{~mm}$ square).

The model parameters used in the simulation of the ceramic failure are provided below in Table 2. 
Table 2: Values used to model the failure of Sintox-FA.

\begin{tabular}{lll}
\hline Description & Variable & Value \\
\hline Reference Density & $\rho_{0}\left(\mathrm{Kg} / \mathrm{m}^{3}\right)$ & 3694 \\
Reference Bulk Modulus & $K_{0}(\mathrm{GPa})$ & 195 \\
Reference Shear Modulus & $G_{0}(\mathrm{GPa})$ & 124 \\
Reference Yield Strength & $Y_{0}(\mathrm{GPa})$ & 3.31 \\
Maximum Yield Strength & $Y_{\max }(\mathrm{GPa})$ & 7.70 \\
Spall Strength & $P_{\mathrm{sp}}(\mathrm{GPa})$ & 0.10 \\
Minimum Yield Factor & $\psi$ & 0.05 \\
Reference Young's Modulus & $E(\mathrm{GPa})$ & 308 \\
Poisson's Ratio & $v$ & 0.22 \\
Coefficient of Friction & $\mu$ & 0.72 \\
Effective Plastic Strain $(1)$ & $\varepsilon_{\mathrm{P} 1}$ & 0.03 \\
Effective Plastic Strain $(2)$ & $\varepsilon_{\mathrm{P} 2}$ & 0.07 \\
Material Constant & $H$ & 1.69 \\
Static Fracture Toughness & $K_{I c}(\mathrm{MPa} \sqrt{\mathrm{m}})$ & 4.60 \\
Longitudinal Wave Velocity & $c_{\mathrm{l}}(\mathrm{km} / \mathrm{s})$ & 9.89 \\
Shear Wave Velocity & $c_{\mathrm{S}}(\mathrm{km} / \mathrm{s})$ & 5.80 \\
\hline
\end{tabular}

An erosion algorithm was introduced to overcome difficulties in mesh tangling during the large deformation of the Lagrangian projectile and target. When the geometric strain of the cell exceeds a specific value, the cell is discarded and its mass is averaged over the surrounding contact nodes. If the erosion strain is set too low, cells will erode prematurely leading to unnecessary energy errors. If the strain is set to a high value, the Lagrangian cells may become distorted, inverted or even tangle. The value chosen in this numerical programme was $200 \%$.

A line drawing of the initial numerical mesh set-up is shown below in Figure 6. 
Figure 6: A line drawing of the initial mesh set-up showing the steel sphere projectile, ceramic target and steel constraining system.

A steel confinement was applied to the ceramic block. During the experiments, this contained the ceramic block.

The model was tested parametrically for sensitivity ${ }^{40}$. This analysis demonstrated that the greatest observed effect was due to changing the material strength parameters. Increasing the strength of the pre-fractured material substantially reduced the penetration depth of the projectile. Changing the values of the coefficient of friction or the ratio of the dynamic to static energy release rate $(h)$ had very little effect on the result.

In addition, the failure patterns that were formed on impact were particularly sensitive to the strength of the material. Increasing the spall strength to $0.3 \mathrm{GPa}$ and $0.5 \mathrm{GPa}$ respectively, reduced the degree of fracture but had little effect on the reduction of the penetration depth. Moreover, the formation of conical type cracks was suppressed. Increasing the strength of the material by $20 \%$ throughout the pressure range of interest (maintaining spall strength of $0.1 \mathrm{GPa}$ ) also suppressed conical crack formation and led to the growth of two large axial cracks extending outward from the target centre.

Changing the initial microcrack angle by $\pm 20 \%$ had little effect on penetration depth and crack formation. This provided validity to the technique of using a fixed angle to 
simulate a problem that consisted of a number of randomly distributed, sized and angled flaws.

The results from an impact by a steel sphere at 1500m/s are shown below in Figure 7. In each case the degree of crack propagation is characterised by the shade of the colours.

Figure 7: Simulated failure of a $25 \mathrm{~mm}$ thick alumina target impacted by a steel sphere at $1500 \mathrm{~m} / \mathrm{s}$.

At $8 \mu$ s from the point of contact between projectile and target, an outer cone (primary) and inner cone crack (secondary) have formed at apex angles of $104^{\circ}$ and $57^{\circ}$ respectively. Lateral cracks have extended outward as the projectile fails and erodes.

By $10 \mu$ s, the lateral cracks have propagated further. Propagation of the cone cracks is also observed. Moreover, another crack propagates from the nucleation site of the primary cone crack. The lateral cracks are seen to curve and propagate towards the surface. At $12 \mu$ s, after impact, failure occurs under the umbrella of the secondary cone crack. The cracks that are formed are not conical in nature. Instead, they are as result of a dynamic complex state of stress that exists under the damage umbrella due to stress wave reflections. By $14.0 \mu$ s the cracks have grown and the stiffness and strength has degraded accordingly.

The simulation was allowed to run to $20 \mu$ s at which point the failure patterns that have evolved were compared to experimental firings. Figure 8 shows the comparison 
between experimental and simulated failure caused by a steel sphere impacting an alumina target at $1500 \mathrm{~m} / \mathrm{s}$. One striking difference is the geometry of the crater, however this is caused by the fact that separation of the Lagrangian mesh is limited. Instead, full crater particulars can be taken from examination of the lateral crack propagation, which, during the experimental firings, cause the front surface of the ceramic to spall.

All major failure phenomena were predicted by the numerical model, including spall, lateral and conical (Hertzian) crack patterns. The model also correctly predicted penetration depths and crater diameters.

\section{PHOTOGRAPH SOMEWHERE HERE (ABOVE NUMERICAL RESULT)}

Figure 8: Result from a numerical simulation of a steel sphere impacting a 25mm Sintox-FA block held in a steel confinement frame $(\mathrm{DOP}=5.8 \mathrm{~mm})$.

The failure of a ceramic target subjected to impact velocities in the range of $1000 \mathrm{~m} / \mathrm{s}$ to $2500 \mathrm{~m} / \mathrm{s}$ was modelled using the developed crack softening model. The penetration of a $6.35 \mathrm{~mm}$ diameter steel sphere into a $25 \mathrm{~mm}$ thick alumina (Sintox-FA) was simulated and compared with experimental results.

Good agreement was predicted for both the measured depth of penetration and the crater diameter. A comparison between the experimental and numerical simulation results for a projectile impacting a 25mm Sintox-FA block are presented in Figure 9 and Figure 10. 
Figure 9: Comparison between the experimental and the numerical depth of penetration measurements for Sintox-FA targets.

The depth of penetration measurements were taken from the centre bottom of the crater. The crater diameter was determined by examining the cracking pattern and estimating the degree of fragmentation (and hence spall) on the surface.

Figure 10: Comparison between the experimental and the numerical crater diameter measurements for Sintox-FA targets.

\subsection{Conclusions}

A physical failure model that describes the processes of crack nucleation, growth and coalescence in compression and tension has been developed and implemented within a numerical hydrocode, AUTODYN-2D. Failure is controlled by the activation of inclined flaws or by the nucleation of fracture by plasticity. In compression, the energy release rate is calculated for the tensile propagation of 'wing' cracks from an inclined flaw and modified to take into account dynamic effects. In tension, the energy release rate is calculated according to time dependent, dynamic fracture mechanics. Crack growth occurs at a velocity dependent on the mode I stress intensity factor. Coalescence and bulk failure of the target occurs when the crack propagates past a cell boundary. The degradation of the yield strength and elastic moduli within a cell is dependent on the size of the crack in relation to the geometry of the cell. 
The model has been used to simulate failure in a dynamically loaded ceramic target over a variety of impact velocities. Good agreement was observed in the penetration depth, crater diameter and fracture patterns for a steel sphere penetrating a confined alumina target. The model predicted the formation of the characteristic failure patterns that occur when a steel sphere penetrates a ceramic target.

\subsection{References}

${ }^{1}$ J. Lankford, Compressive Strength and Microplasticity in Polycrystalline Alumina. J. Materials Science, 12, 791-796 (1977).

${ }^{2}$ D. C Larsen, J. W. Adams, S. A. Bortz and R. Ruh, Evidence of Strength Degradation by Subcritical Crack Growth in Si3N4 and SiC. Proceedings of the International Symposium on the Fracture Mechanics of Ceramics, In Fracture Mechanics of Ceramics, Plenum Press, New York, 571-585 (1983).

${ }^{3}$ AUTODYN ${ }^{\mathrm{TM}}$ Theory Manual, Century Dynamics Ltd. Dynamics House, Hurst Road, Horsham, West Sussex, RH12 2DT, UK (1991).

${ }^{4}$ P. J. Hazell and M. J. Iremonger, Crack Softening Damage Model for Ceramic Impact and its Application within a Hydrocode. J. Appl. Phys. 82, (3) 1088-1092 (1997).

${ }^{5}$ L. G. Margolin, Elastic Moduli of a Cracked Body. Int. J. of Fract. 22, 65-79 (1983).

${ }^{6}$ L. S. Costin and C. M. Stone, A Finite Element Material Model for Microfracture Damaged Brittle Rock. Sandia Technical Report SAND87-1227, UC-13 (1987).

${ }^{7}$ H. Horri and S. Nemat-Nasser, Brittle Failure in Compression: Splitting, Faulting and Brittle-Ductile Transition. Phil. Trans. R. Soc. Lond. 319, 337-374 (1986).

${ }^{8}$ D. Krajcinovic and D. Fanella, A Micromechanical Model for Concrete. Engng. Fracture Mech. 25, (5/6) 585-596 (1986).

${ }^{9}$ R. Ilankamban and D. Krajcinovic, A Constitutive Theory for Progressively Deteriorating Brittle Solids. Int. J. Solids Structures, 23, (7) 1521-1534 (1987).

${ }^{10}$ G. Ravichandran and G. Subhash, A Micromechanical Model for High Strain Rate Behaviour of Ceramics. Int. J. Solids and Structures, 32, (17/18) 2627-2646 (1995). 
${ }^{11}$ H. Deng and S. Nemat-Nasser, Dynamic Damage Evolution in Brittle Solids. Mech. Mater. 14, 83-103 (1992).

${ }^{12}$ W. F. Brace and E. G. Bombolakis, A Note on Brittle Crack Growth in Compression. J. Geophys. Res. 68, 3709-3713 (1963).

${ }^{13}$ F. L. Addessio and J. N. Johnson, A Constitutive Model for the Dynamic Response of Brittle Materials. J. Appl. Phys. 67, (7) 3275-3286 (1990).

${ }^{14}$ A. M. Rajendran, Modeling the Shock Response of AD995 Alumina. High Pressure Science and Technology - 1993, Part 2, AIP Press, New York, 725-728 (1994).

${ }^{15}$ C. J. Hayhurst and R. A. Clegg, Cylindrically Symmetric SPH Simulations of Hypervelocity Impacts on Thin Plates. Int. J. Impact Engng, 20, 337-348 (1997).

${ }^{16}$ D. A. Mandell and C. A. Wingate, Prediction of Material Strength and Fracture of Glass Using the SPHINX Smooth Particle Hydrodynamics Code, Los Alamos Technical Report LA-12830, Los Alamos National Laboratory, New Mexico, USA, August (1994).

${ }^{17}$ D. R. J. Owen, A. J. L. Crook, J-G. Yu and M. Dutko, Characterisation of the Material Failure and Fracture Propagation in Brittle Materials subjected to High Strain Rates. Lightweight Armour Systems Symposium, Royal Military College of Science, Shrivenham, UK, 12th-14th November (1997).

${ }^{18}$ H. Horri and S. Nemat-Nasser, Compression-Induced Microcrack Growth in Brittle Solids: Axial Splitting and Shear Failure. J. Geophys. Res. 90, (B4) 3105-3125 (1985).

${ }^{19}$ G. Venkins, M. F. Ashby and P. W. R. Beaumont, The Compressive Failure of Alumina Containing Controlled Distributions of Flaws. Acta Metall. Mater. 39, (11) 2583-2588 (1991).

${ }^{20}$ M. F. Ashby and S. D. Hallam, The Failure of Brittle Solids Containing Small Cracks Under Compressive Stress States. Acta Metall. Mater. 34, (3) 497-510 (1986).

${ }^{21}$ M. F. Ashby and C. G. Sammis, The Damage Mechanics of Brittle Solids in Compression. PAGEOPH, 133, (3) 489-521 (1990).

${ }^{22}$ F. Longy and J. Cagnoux, Plasticity and Microcracking in Shock-Loaded Alumina. J. Am. Ceram. Soc. 72, (6) 971-979 (1989). 
${ }^{23}$ B. J. Hockey and S. M. Wiederhorn, Erosion of Ceramic Materials: The Role of Plastic Flow. In the Proceedings of the 5th International Conference on Erosion by Solid and Liquid Impact, (26) pp 1-9 (1979).

${ }^{24}$ A. Persson, CM1 - A Simple Model for the Dynamic Deformation and Failure Properties of Brittle Materials. In the Proceedings of the 4th International Symposium in Ceramic Materials and Components for Engines, Ed. R. Carlsson, T. Johansson and L. Kahlman, 953-959 (1990).

${ }^{25}$ L. B. Freund, Dynamic Fracture Mechanics, Cambridge University Press, Cambridge, 1990.

${ }^{26}$ S. Nemat-Nasser and H. Deng, Strain-Rate Effect on Brittle Failure in Compression. Acta Metall. Mater. 42, (3) 1013-1024 (1994).

${ }^{27}$ K. Kishida, T. Yokoyama and M. Nakano, Measurement of the Dynamic Fracture Toughness Based on the Split Hopkinson Bar Technique. Proc. 3rd Conf. Mech. Prop. High Rates of Strain, Inst. Phys. Conf. Ser. 70, 221-228 (1984).

${ }^{28}$ J. Duffy, T. Nakamura, Y. Yeshurun and S. Suresh, Dynamic Fracture of Ceramics. Proc. 4th Conf. Mech. Prop. High Rates of Strain, Inst. Phys. Conf. Ser. 102, 355362 (1989).

${ }^{29}$ D. A. Shockey, J. F. Kalthoff and D. C. Erlick, Evaluation of Dynamic Crack Instability Criteria. Int. J. of Fract. 22, 217-229 (1983).

${ }^{30}$ K. Yang and A. S. Kobayashi, Dynamic Fracture Responses of Alumina and Two Ceramic Composites. J. Am. Ceram. Soc. 73, (8) 2309-2315 (1990).

${ }^{31}$ J. W. Dally, W. L. Fourney and G. R. Irwin, On the Uniqueness of the Stress Intensity Factor - Crack Velocity Relationship. Int. J. of Fract. 27, 159-168 (1985).

${ }^{32}$ S. Suresh, T. Nakamura, Y. Yeshurun, K, H, Yang and J. Duffy, Tensile Fracture Toughness of Ceramic Materials: Effects of Dynamic Loading and Elevated Temperatures. J. Am. Ceram. Soc. 73, (8) 2457-2466 (1990).

${ }^{33}$ W. Fenghui, Z. Xiulin and L. Mingxu, On the Strength of Ceramics with Cavities. Int. J. Fract. 70, R19-R22 (1995).

${ }^{34}$ R. L. Coble and W. D. Kingery, Effect of Porosity on Physical Properties of Sintered Alumina. J. Am. Ceram. Soc. 39, (11) 377-385 (1956).

${ }^{35}$ M. J. Hallas, Shattered Ceramic and their Effectiveness as Armour. No. 1 Defence Technology Course, MSc Report, Royal Military College of Science, Shrivenham, UK (1997). 
${ }^{36}$ C. J. Coakley, Modelling of Long Rod Penetration into Alumina Ceramic, Defence Research Agency Report DRA/FVS/AM/CR94012/1.0, UK October (1994).

37 J. Cagnoux and F. Longy, Is the Dynamic Strength of Alumina Rate Dependent? In Shock Waves in Condensed Matter. Ed S. C. Schmidt and N. C. Holmes, Elsevier Science Publications, 293-296 (1988).

${ }^{38}$ H. C. Heard and C. F. Cline, Mechanical Behaviour of Polycrystalline $\mathrm{BeO}, \mathrm{Al}_{2} \mathrm{O}_{3}$ and AlN at High Pressure. Journal of Material Science, 15, 1889-1897 (1980).

${ }^{39}$ B. James, The Influence of the Material Properties of Alumina on Ballistic Performance. Defence Research Agency Report DRA/FV\&S/AM/TR94003/1.0, Farnborough, UK, May (1994).

${ }^{40}$ P. J. Hazell, The Failure of Ceramic Armour Subjected to High Velocity Impact. EngD Thesis, Cranfield University, Royal Military College of Science, UK (1998). 


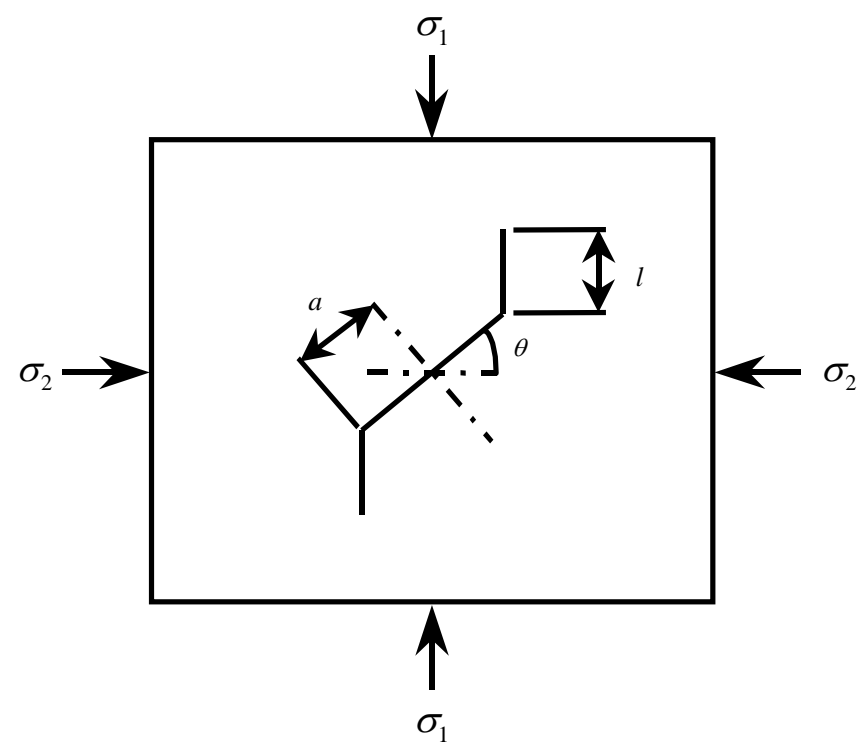

Figure 1: Idealised unit cell model for a sliding crack under biaxial compression. 


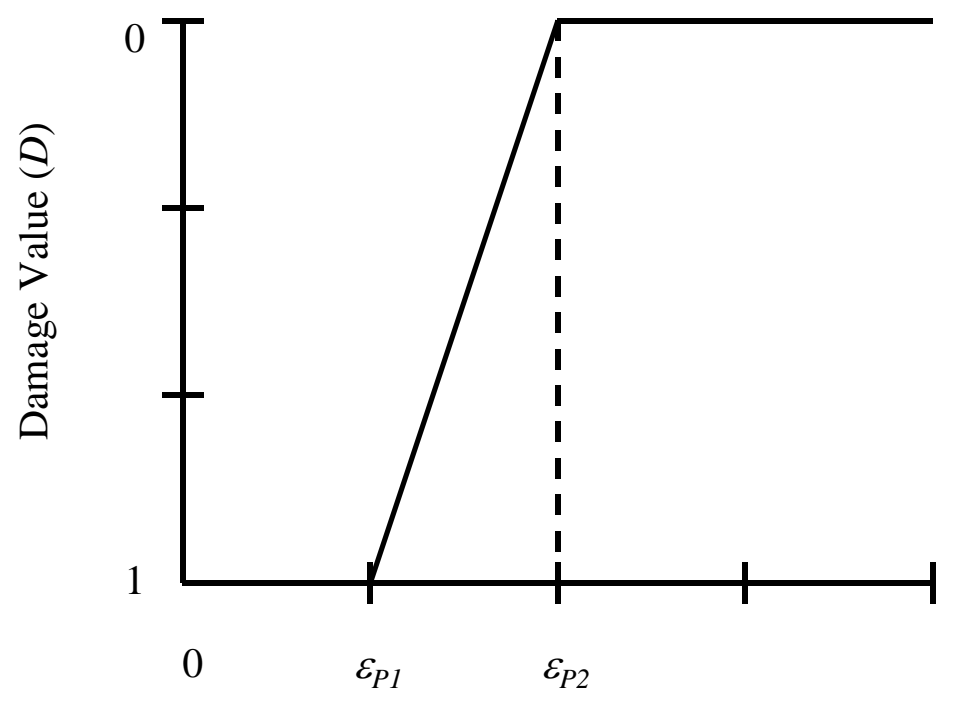

Effective Plastic Strain

Figure 2: Relationship between the effective plastic strain and the damage accumulated within a Lagrangian cell ( $D=1$ represents the intact material, $D=0$ represents failed material). 


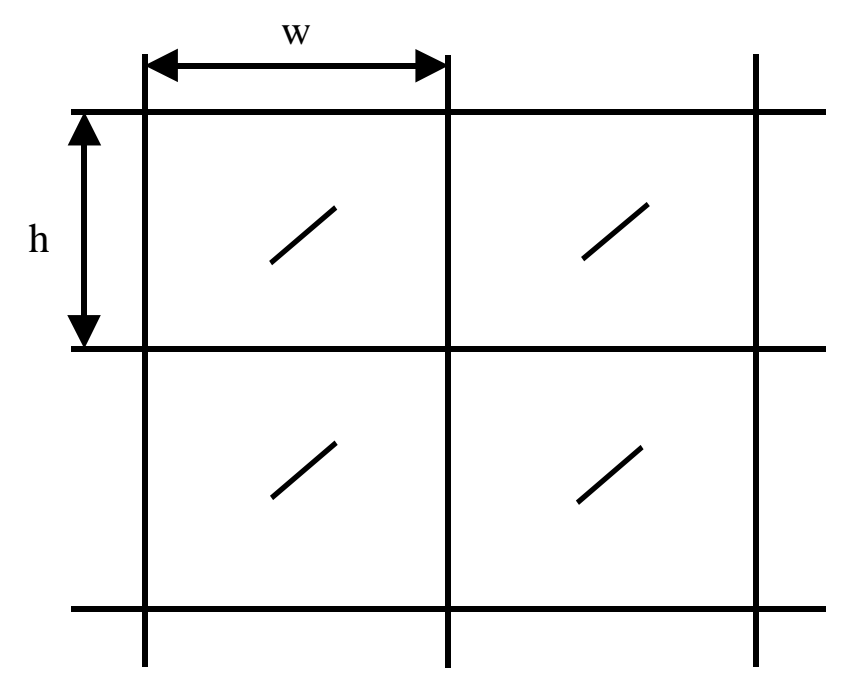

Figure 3: Initial distribution of flaws in cells of height $\mathrm{h}$ and width $\mathrm{w}$. 


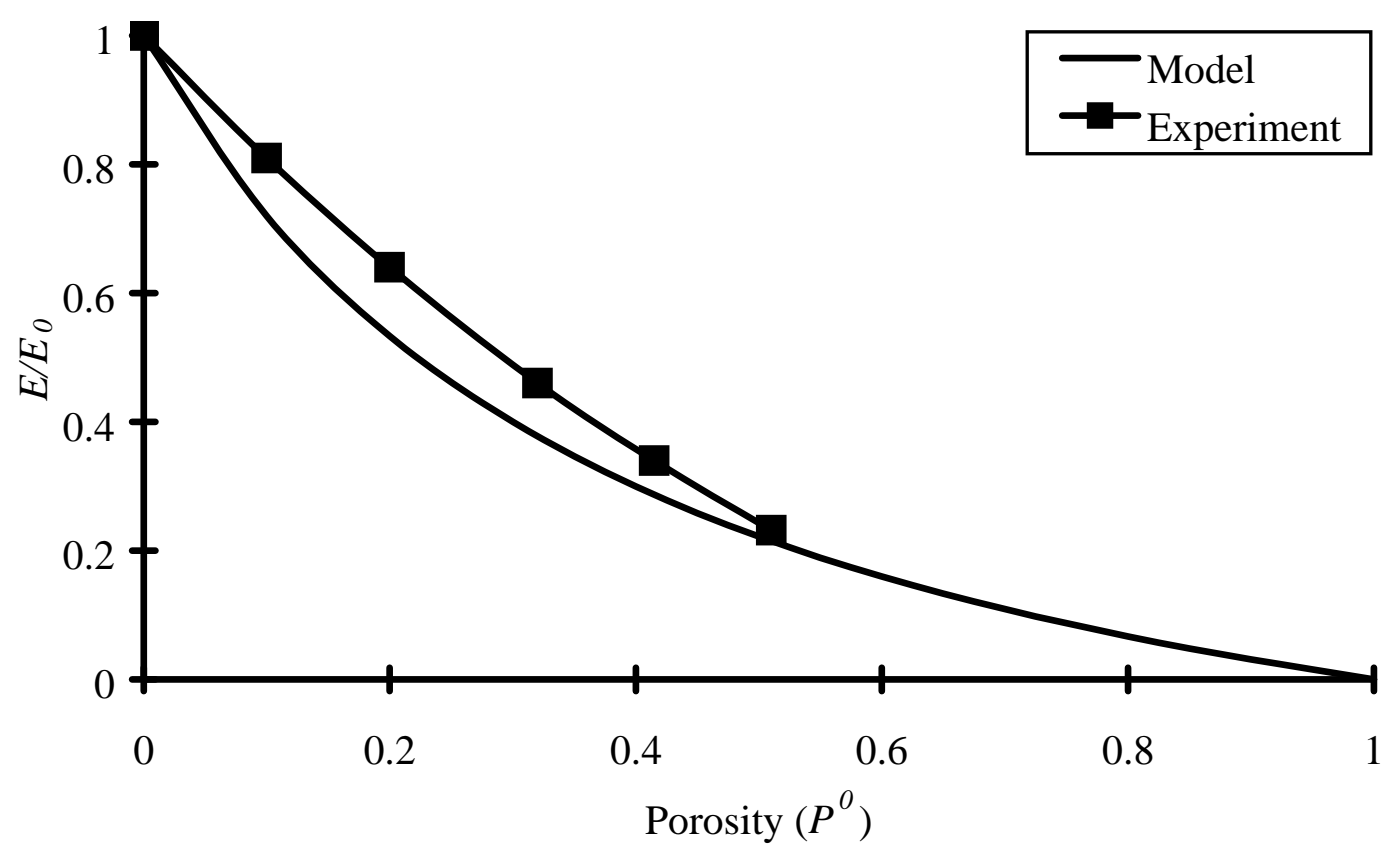

Figure 4: Degradation of the elastic moduli with increasing porosity. 


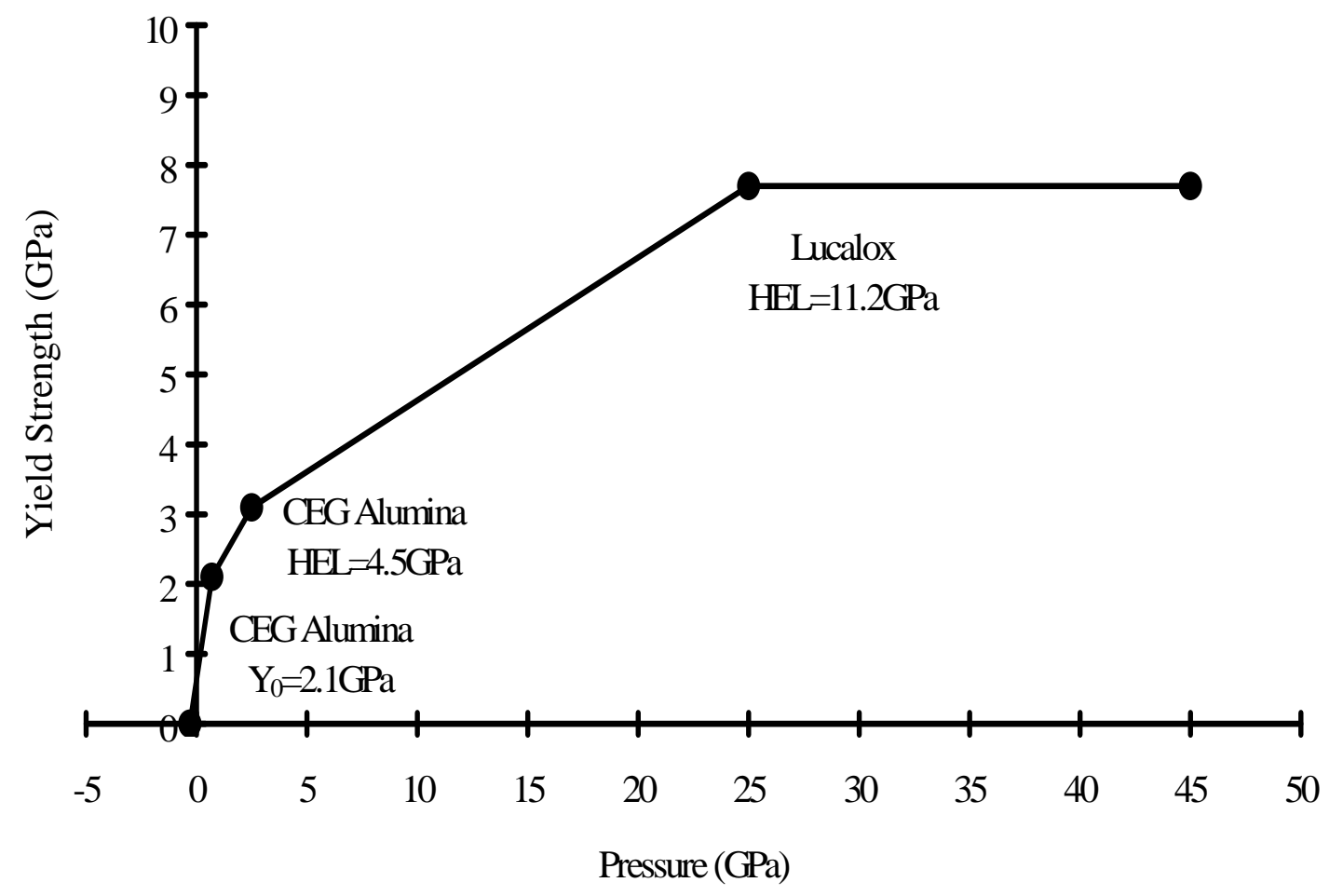

Figure 5: A Pressure dependent yield strength relationship for alumina (after Ref. 36). 
Projectile

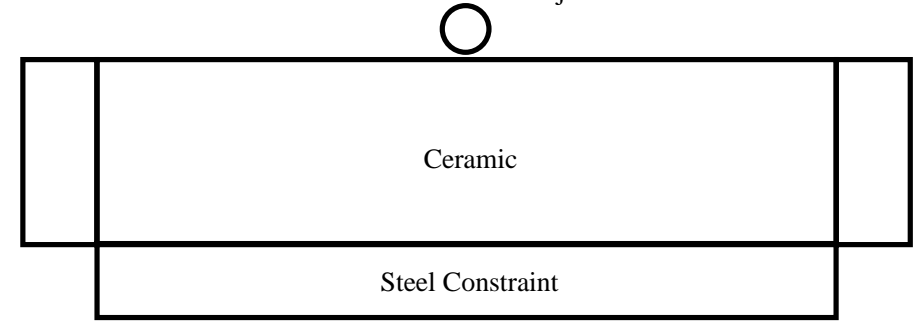

Figure 6: A line drawing of the initial mesh set-up showing the steel sphere projectile, ceramic target and steel constraining system. 

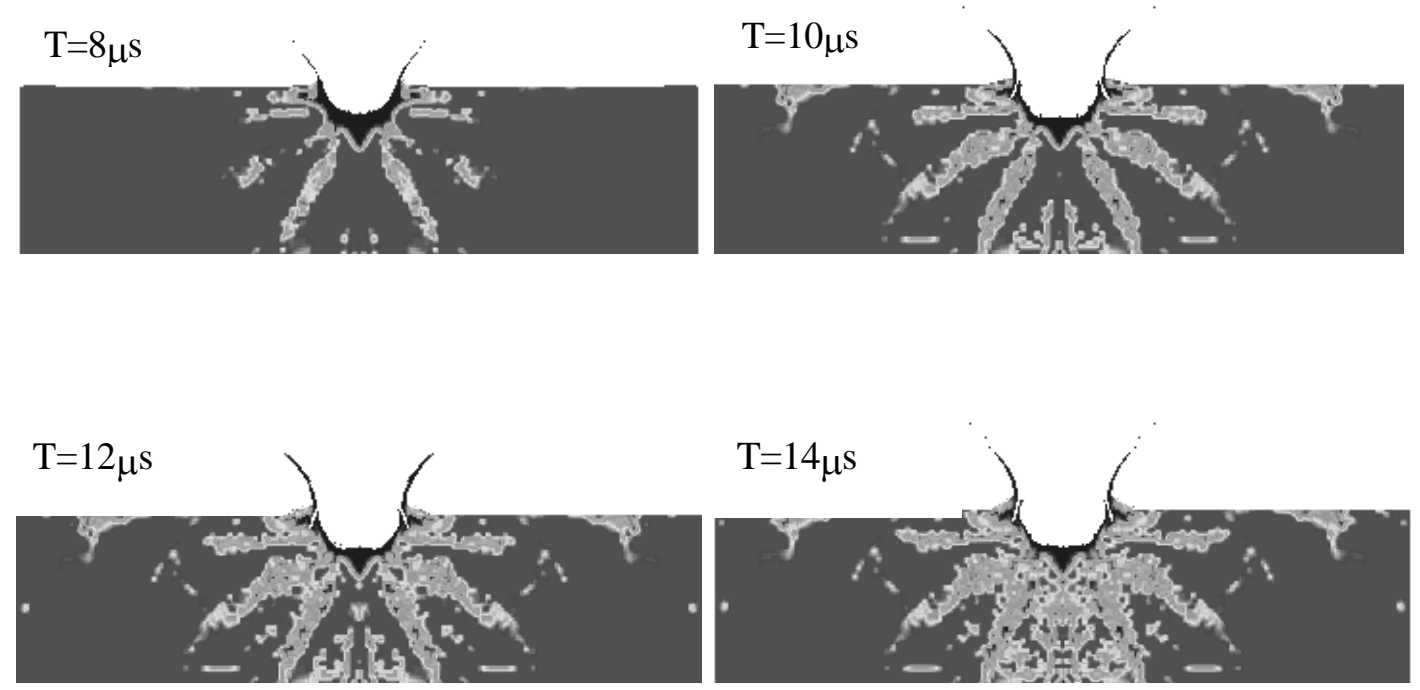

Figure 7: Simulated failure of a $25 \mathrm{~mm}$ thick alumina target impacted by a steel sphere at $1500 \mathrm{~m} / \mathrm{s}$. 

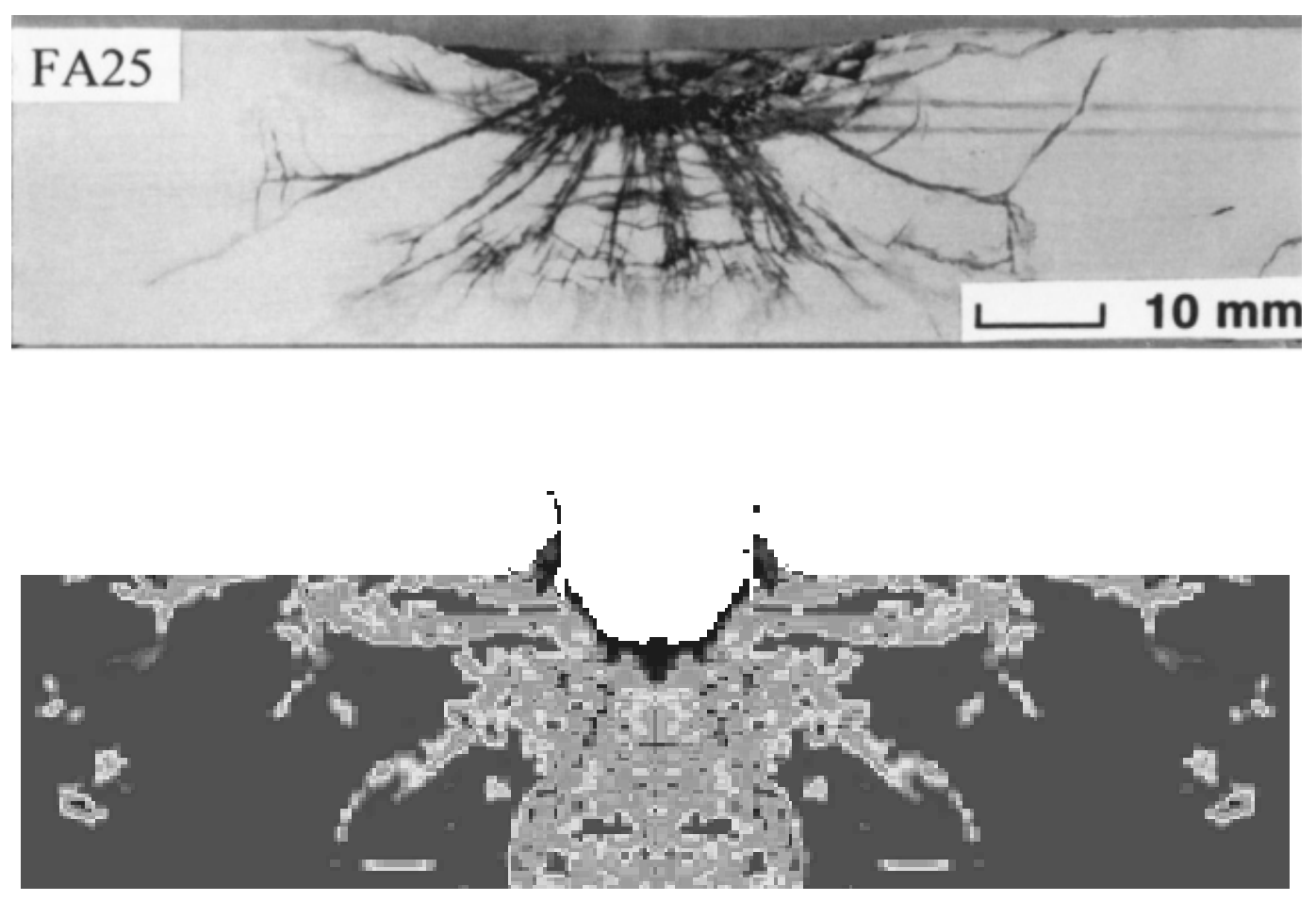

Figure 8: Result from a numerical simulation of a steel sphere impacting a 25mm Sintox-FA block held in a steel confinement frame $(\mathrm{DOP}=5.8 \mathrm{~mm})$. 


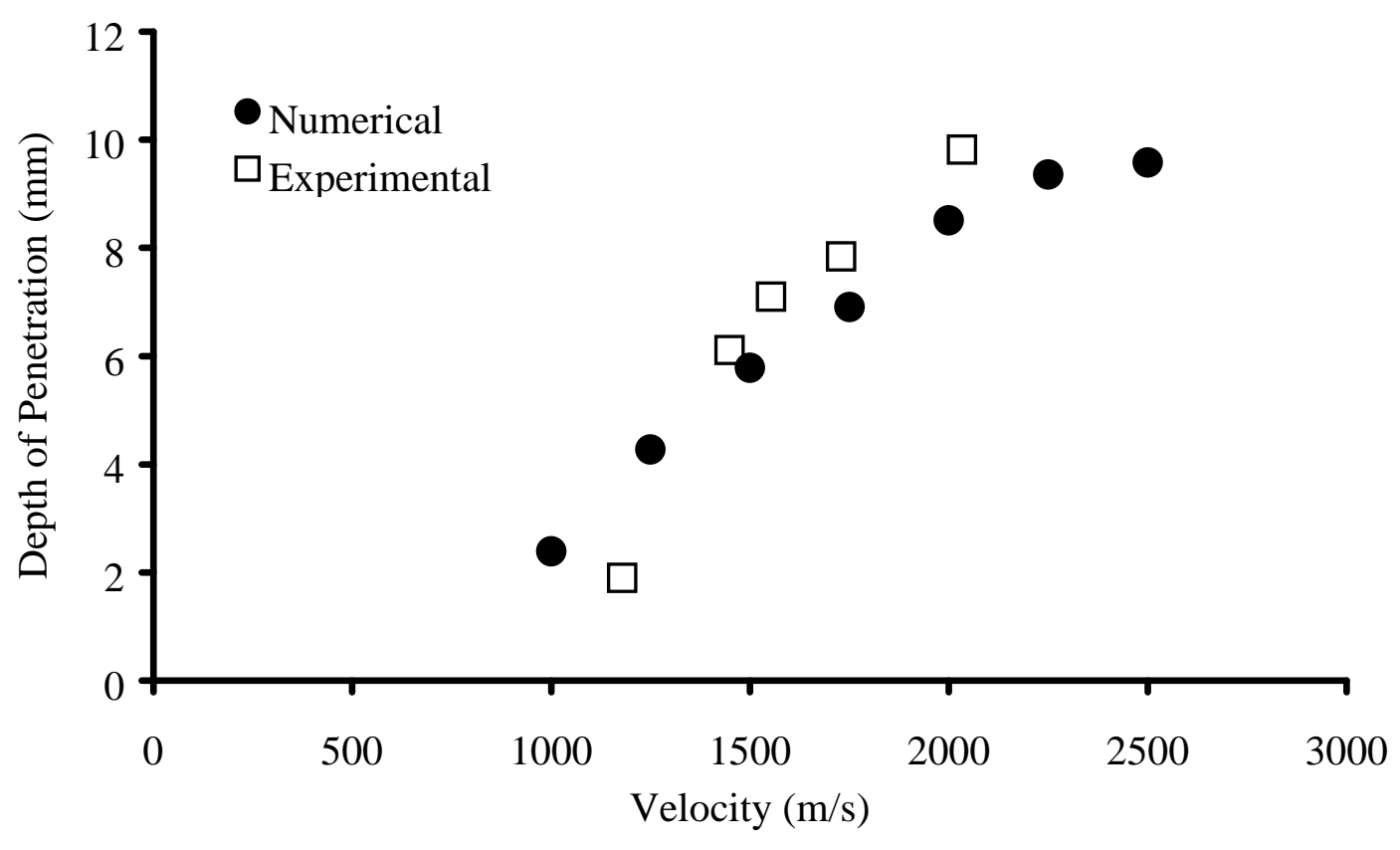

Figure 9: Comparison between the experimental and the numerical depth of penetration measurements for Sintox-FA targets. 


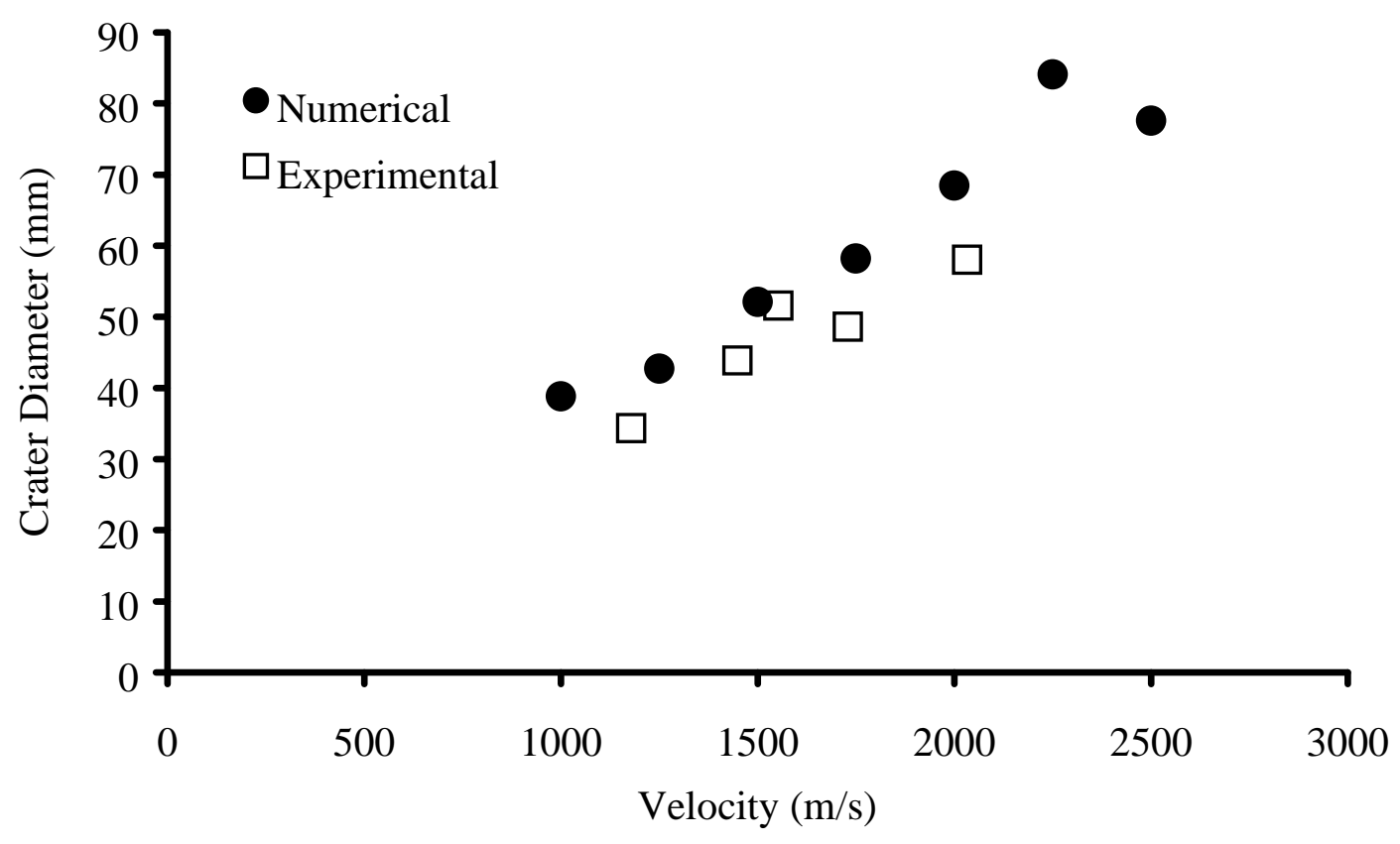

Figure 10: Comparison between the experimental and the numerical crater diameter measurements for Sintox-FA targets. 